\title{
Fatty acyl availability modulates cardiolipin composition and alters mitochondrial function in HeLa cells
}

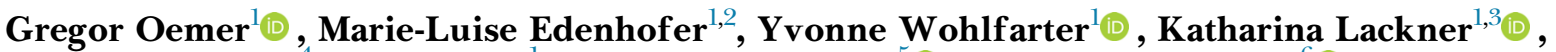 \\ Geraldine Leman ${ }^{4}$, Jakob Koch ${ }^{1}$, Luiza H. D. Cardoso ${ }^{5}(0)$, Herbert H. Lindner ${ }^{6}$, Erich Gnaiger ${ }^{5}$, \\ Sandrine Dubrac ${ }^{4}\left(\mathbb{D}\right.$, Johannes Zschocke ${ }^{1}\left(\mathbb{D}\right.$, and Markus A. Keller ${ }^{1, *(\mathbb{D})}$ \\ ${ }^{1}$ Institute of Human Genetics, ${ }^{2}$ Institute of Physiology, ${ }^{3}$ Institute of Biological Chemistry, Biocenter Innsbruck, ${ }^{4}$ Epidermal \\ Biology Laboratory, Department of Dermatology, Venereology and Allergology, Medical University of Innsbruck, \\ Innsbruck, Austria; ${ }^{5}$ Oroboros Instruments Corporation, Innsbruck, Austria; and ${ }^{6}$ Institute of Clinical Biochemistry, Medical \\ University of Innsbruck, Innsbruck, Austria
}

\begin{abstract}
The molecular assembly of cells depends not only on the balance between anabolism and catabolism but to a large degree on the building blocks available in the environment. For cultured mammalian cells, this is largely determined by the composition of the applied growth medium. Here, we study the impact of lipids in the medium on mitochondrial membrane architecture and function by combining LC-MS/MS lipidomics and functional tests with lipid supplementation experiments in an otherwise serum-free and lipid-free cell culture model. We demonstrate that the composition of mitochondrial cardiolipins strongly depends on the lipid environment in cultured cells and favors the incorporation of essential linoleic acid over other fatty acids. Simultaneously, the mitochondrial respiratory complex I activity was altered, whereas the matrix-localized enzyme citrate synthase was unaffected. This raises the question on a link between membrane composition and respiratory control. In summary, we found a strong dependency of central mitochondrial features on the type of lipids contained in the growth medium. Fir This underlines the importance of considering these factors when using and establishing cell culture models in biomedical research. In summary, we found a strong dependency of central mitochondrial features on the type of lipids contained in the growth medium.
\end{abstract}

Supplementary key words lipids $\cdot$ FAs $\cdot$ MS • cardiolipin • mitochondria

Immortalized human cell lines are widely used models for biochemistry, cell biology, and human diseases and used in many research laboratories worldwide. They have evaded natural senescence by accumulation of proliferating mutations and can therefore be grown for prolonged periods of time in vitro (1). However, they require a variety of essential nutrients that are supplied with the cell culture medium. Different cell lines require

*For correspondence: Markus A. Keller, markus.keller@i-med.ac.at.
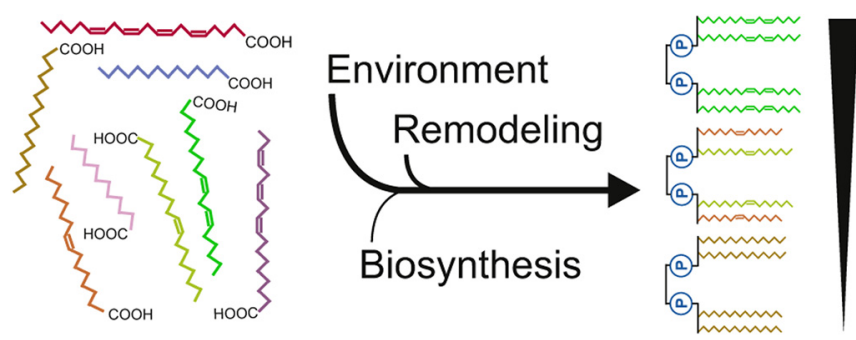

different medium compositions to be viable and proliferate. For HeLa cells, the first published immortalized cell line derived from cervical cancer cells of Henrietta Lacks in 1951 (2), Harry Eagle developed his MEM (3), which was modified by Renato Dulbecco in 1959 (DMEM) (4) consisting of inorganic salts, amino acids, vitamins, Dglucose, sodium pyruvate, and phenol red and is now one of the most used cell culture media (5). In addition, also nonsynthetic components are added, of ten derived from FBS, which contains a complex mixture of proteins, growth factors, as well as lipids and is added to the medium before usage.

To avoid batch-to-batch variation, to promote animal-free cell culture, but also to reduce costs, serumfree and chemically defined media have been developed (6). Nevertheless, serum-containing growth conditions are still heavily used in many research laboratories. Obviously, the composition of cell culture media affects cellular composition, metabolism, proliferation, and other functions. The impact of different media components on cellular functions has been studied with variable intensity. In particular, the lipid content of the medium has received little attention in the development of recent decades. For example, most cell culture media, such as MEM, DMEM, and RPMI, do not contain any lipids, and the only external source is nonchemically defined FBS, even though essential fatty acids cannot be synthesized de novo by human cells, 
and their dietary availability has substantial impact on health and disease (7).

This lack of lipid content-related optimization also manifests itself in the addition of plant lipid extracts or the like in some synthetic media, which are in their fatty acid composition not necessarily matched for mimicking the original environment of mammalian cells. In previous work, we could demonstrate that HeLa cells supplemented with heart lipid extract exhibited strong lipid profile changes of mitochondrial membrane phospholipids causing a lowered basal respiration while respiratory complex I (CI) activity was increased (8). This shows that an uncontrolled composition of lipids in the cell culture medium can lead to significant functional changes. As we have recently demonstrated, in mitochondrial membranes, the lipid class of cardiolipins (CLs) is especially responsive to the fatty acyl environment $(9,10)$.

CLs are dimeric phospholipids with four fatty acyl side chains and are exclusively found in mitochondria. CLs have a strong preference for the inner mitochondrial membrane where they are synthesized and make up $20 \%$ of the total phospholipid content (11). The unique structure of CLs allows them to be functionally involved in mitochondrial bioenergetics $(11,12)$. This includes the conical shape of CL that assist with cristae structure formation, the interaction of cristae junctions with different protein complexes (13), and control of mitochondrial dynamics $(14,15)$. Furthermore, direct binding between CLs and respiratory chain complexes is key to (super-)complex formation and stabilization (16-18). CLs are also potent reactive oxygen species
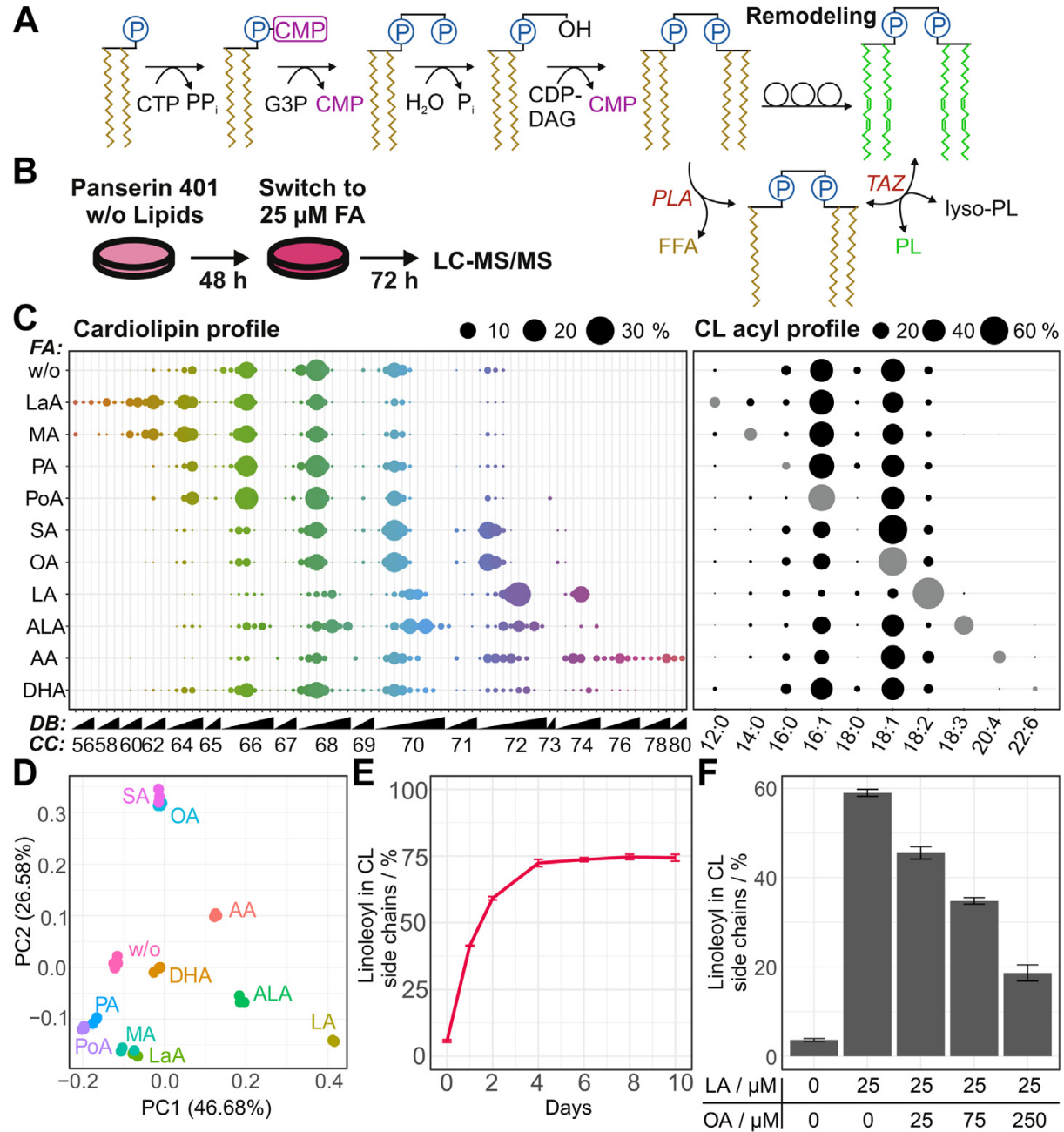

Fig. 1. Fatty acid supplementation leads to strong fatty acyl substitution changes in CL compositions. A: CL biosynthesis and remodeling process. B: Schematic depiction of cell culture experiments. C: Mean CL profile (right) of HeLa (Mol-\%) cultivated in FBS-free panserin 401 without lipids and after supplementation for $72 \mathrm{~h}$ with $25 \mu \mathrm{M}$ fatty acid and their respective relative CL acyl profile (left), which was obtained by utilizing MS/MS fragment spectra and mathematical modeling. DB: total double bonds; CC: total carbon chain length; gray color: FA supplemented in each respective experiment. Mean \pm SD profiles are shown in supplemental Figs. S2 and S3. D: principal component analysis (PCA) of CL profile showing a clear separation of supplemented conditions. Centered relative CL abundances were used for this analysis explaining over 70\% of variation in the first two dimensions (PC1 and PC2). E: LA incorporation reaches a steady-state plateau after 3-4 days in proliferating HeLa. F: LA incorporation is clearly preferred over OA but attenuated at high excess of the latter. 
scavengers, a process that leads to CL externalization to the outer mitochondrial membrane, where they recruit proapoptotic proteins resulting in the release of cytochrome $c$ (cyt $c$ ) into the cytosol and consequently apoptosis $(19,20)$ or mitophagy $(21,22)$. Importantly, the acyl side chain composition of CL is not controlled by substrate specificity of their biosynthesis but requires additional enzymatic remodeling processes (Fig. 1A) (23, 24). In total, three enzymes have the ability to remodel CL, but only one-tafazzin-is so far reported to be pathogenic when impaired. Notably, CL compositions vary strongly not only across species (8) but also between tissues within one organism (10). Furthermore, a clear dependency of the CL composition and nutritionally available lipids is known (25). In addition to the aforementioned stated observations, such CL compositions have been shown to affect certain enzymatic specificities such as the cyt $c$ peroxidase function, which has a higher affinity toward polyunsaturated CLs (26).

Here, we address the question to which extend externally added fatty acids impact on CL compositions in an otherwise lipid-free environment. We investigate the speed by which the mitochondrial membrane lipid compositions adapt to changes in the fatty acyl environment and how these changes influence mitochondrial functions. To tackle this, we use a completely controlled serum-free and lipid-free cell culture model for HeLa, which can be readily supplemented with a set of fatty acids and heart lipid extract.

\section{MATERIALS AND METHODS}

\section{Cell culture}

If not stated otherwise, all cells were grown at $37^{\circ} \mathrm{C}, 100 \%$ humidity, and in an atmosphere of $5 \% \mathrm{CO}_{2}$. HeLa cells were maintained in $25 \mathrm{~cm}^{2}$ flasks in DMEM, $1 \mathrm{~g} / 1$ D-glucose, and sodium bicarbonate (D5546; Sigma-Aldrich, St. Louis, MA) supplemented with $10 \%(\mathrm{v} / \mathrm{v})$ heat-inactivated fetal calf serum (10500-064; Gibco, Invitrogen, Carlsbad, CA), 2 mM L-glutamine (17-605E; Lonza Group, Basel, Switzerland), and $100 \mathrm{U} /$ $\mathrm{ml}$ penicillin-streptomycin (17-602E; Lonza Group, Basel, Switzerland). For serum- and lipid-free cell culture, medium was exchanged to customized panserin 401 without lipids (Pan-Biotech, Aidenbach, Germany) for at least 2-3 days before further treatment.

\section{Fatty acid-BSA conjugates}

For lipid supplementation, fatty acid-BSA complexes were generated as described (8). Briefly, fatty acids were weighed and dissolved as a $100 \mathrm{mM}$ stock in $100 \mathrm{mM} \mathrm{NaOH}$. The mixture was incubated at $70^{\circ} \mathrm{C}$ (for stearic and palmitic acid $[\mathrm{PA}], 85^{\circ} \mathrm{C}$ ) in a water bath until complete solubilization. Fatty acid-free BSA (A7030; Sigma-Aldrich, St. Louis, MA) was dissolved in cell culture medium $(2.8 \mathrm{mM}$, panserin 401 without lipids), incubated at $37^{\circ} \mathrm{C}$, and thoroughly vortexed until complete solvation. While still warm, the $100 \mathrm{mM}$ fatty acid (FA) solution was diluted 1:5 in the $2.8 \mathrm{mM}$ BSA solution (final concentration: $20 \mathrm{mM} \mathrm{FA}$ in $2.28 \mathrm{mM} \mathrm{BSA}$ ) shaken for $20 \mathrm{~min}$ at $55^{\circ} \mathrm{C}$ at $1,000 \mathrm{rpm}$. This BSA-complexed fatty acid stock solution was added to the cell culture medium for later supplementation (1:800 for $25 \mu \mathrm{M}$ FA in $2.8 \mu \mathrm{M}$ BSA solution). Medium was prepared freshly and placed at $4^{\circ} \mathrm{C}$ for shortterm storage. If not stated otherwise, the medium was exchanged every second day. PUFAs were stored at $-20^{\circ} \mathrm{C}$ in aliquots under an argon atmosphere.

\section{Fatty acid supplementation of HeLa cells}

For fatty acid supplementation experiments, 0.25 million cells, grown under standard conditions, were split in six wells with panserin 401 without lipids and incubated for 2 days. Then, the medium was exchanged to panserin 401 containing $25 \mu \mathrm{M}$ of the respective supplemented fatty acid for $72 \mathrm{~h}$. Cells were harvested by trypsinization, washed with PBS, and pelleted by centrifugation. Cell pellets were stored at $-20^{\circ} \mathrm{C}$ for subsequent HPLC-MS/MS analysis. For fatty acid exchange experiments, 0.2 million cells were seeded in a 6 -well plate for $48 \mathrm{~h}$ in panserin 401 without lipids before exchanging the medium to the new target condition. Cells were pelleted as described previously at the respective time points. Analogue, for respirometry, 0.5 million cells were seeded in $75 \mathrm{~cm}^{2}$ flasks for $48 \mathrm{~h}$ in panserin 401 without lipids before exchanging the medium to the new target condition.

\section{Heart lipid supplementation of HeLa cells}

About $20 \mathrm{~g}$ of fresh pig heart (purchased at the local butchery) were homogenized in $500 \mathrm{ml} 1 \times$ PBS using a hand blender (Bosch, Germany). The lipids were extracted following the Folch method (27) using $500 \mathrm{ml}$ 2:1 chloroform/ methanol mixture in a 1 liter separating funnel. Organic phases containing the lipid extract were evaporated in a Rotavapor (Büchei, Flawil, Schweiz) at $40^{\circ} \mathrm{C}$ and 750 mbar. The dried lipid extracts were weighed, to $25 \mathrm{mg} / \mathrm{ml}$ with 2:1 chloroform/methanol mixture and stored in $2 \mathrm{ml}$ aliquots in glass vials at $-80^{\circ} \mathrm{C}$. For heart lipid supplementation, $40 \mathrm{mg}$ heart lipid extract was added to $200 \mathrm{ml}$ lipid-free panserin 401 and shaken in water bath at $50^{\circ} \mathrm{C}$ for $24 \mathrm{~h}(200 \mu \mathrm{g} / \mathrm{ml}$ final concentration). The supplemented medium was sterile filtered through $0.2 \mu \mathrm{m}$ pore filter. HeLa cells were grown in $25 \mathrm{~cm}^{2}$ flasks with serum-free panserin 401 without lipids supplemented with pig heart lipids for 3 days and were then harvested.

\section{HPLC-MS/MS analysis of CLs}

CL analysis was performed as described (8). Briefly, sample material was homogenized in PBS, and lipids were extracted following the Folch method (27) with internal standard $\left(\mathrm{CL}(14: 0)_{4}, 0.5 \mu \mathrm{M}\right)$. Lipid extracts were dissolved in HPLC starting condition and subjected to HPLC-MS/MS analysis. Separation was achieved by reversed-phase HPLC with an Agilent Poroshell 120 EC-C8 $2.7 \mu \mathrm{m} 2.1 \times 100 \mathrm{~mm}$ column (Agilent Technologies, Santa Clara, CA) on a Dionex Ultimate 3000 HPLC (Thermo Fisher Scientific, Inc, Waltham, MA; $50^{\circ} \mathrm{C}$ column oven, $0.4 \mu \mathrm{l} / \mathrm{min}$ flow) with running solvent $\mathrm{A}$ $\left(60 / 40\right.$ acetonitrile $/ \mathrm{H}_{2} \mathrm{O}, 10 \mathrm{mM}$ ammonium formate, and $0.2 \%$ FA) and running solvent B (90/10 isopropanol/acetonitrile, $10 \mathrm{mM}$ ammonium formate, and $0.2 \% \mathrm{FA}$ ). Analytes were measured using a LTQ Velos MS (Thermo Fisher Scientific, Inc, Waltham, MA) operated in negative ESI mode $(3.8 \mathrm{kV}$, $275^{\circ} \mathrm{C}$ capillary temperature, $460-1,650 \mathrm{~m} / \mathrm{z}$ ) and datadependent $\mathrm{MS}^{2}$ acquisition. Thermo raw data were converted to open-source MZML format, and peaks were integrated in MZmine2 (28). Identification was based on a 
combination of accurate mass, (relative) retention times, and fragmentation spectra, compared with a library of standards. Normalization, quantification, and data analysis were performed by an in-house pipeline in $\mathrm{R}$ software ( $\mathrm{R}$ Core Team, https://www.r-project.org/) (29). CL acyl composition was modeled by a two-step calculation: A matrix of all possible CL fragments was aligned to measured $\mathrm{MS}^{2}$ spectra by "BroydenFletcher-Goldfarb-Shanno" minimization. These CL fragments were aligned to a matrix of possible FAs and minimized using the "Broyden-Fletcher-Goldfarb-Shanno" algorithm. Prediction of CL profile with modeled FA and CL fragments was used as quality control. Phospholipids were measured as described (30).

\section{Cell viability}

Cell viability was determined using the Cell Counting Kit-8 (CCK-8; Dojindo Laboratories, Japan) in which, the reagent WST-8 [2-(2-methoxy-4-nitrophenyl)-3-(4-nitrophenyl)-5- (2,4disulfophenyl)-2H-tetrazolium, monosodium salt] was reduced by dehydrogenases active only in viable cells to formazan, an orange soluble dye measurable by a UV/visible system. Via a dilution series of each supplemented fatty acid, the $\mathrm{LD}_{50}$ values were calculated for each fatty acid in HeLa. Briefly, 5,000 cells suspended in $50 \mu \mathrm{l}$ panserin 401 were added to each well in a 96 -well plate. About $50 \mu \mathrm{l}$ of the same medium containing the supplemented fatty acids in different concentrations were then added and incubated for 3 days. Then $10 \mu$ of the CCK- 8 solution were added to each well, and the plate was incubated for $2 \mathrm{~h}$ at $37^{\circ} \mathrm{C}$ and $5 \% \mathrm{CO}_{2}$. Finally, the absorption was measured at $450 \mathrm{~nm}$ by a plate reader (PHERAstar, BMG Labtech, Ortenberg, Germany).

\section{Enzyme activity assays}

For measuring the enzymatic activity of complex I and citrate synthase (CS), mitochondrial-enriched fractions were prepared. Therefore, cell pellets were suspended in $150 \mu \mathrm{l}$ saccharose buffer $(250 \mathrm{mM}$ saccharose, $20 \mathrm{mM}$ Tris, $2 \mathrm{mM}$ EDTA, $1 \mathrm{mg} / \mathrm{ml} \mathrm{BSA}$; $\mathrm{pH}=7.2)$. After one freezing cycle in liquid nitrogen and thawing cycle at $37^{\circ} \mathrm{C}$, the cells were centrifuged at 12,000 rcf for $90 \mathrm{~min}$. The mitochondrial cell pellet was resuspended in $150 \mu \mathrm{l}$ hypotonic buffer $(25 \mathrm{mmol} / \mathrm{l}$ $\mathrm{KH}_{2} \mathrm{PO}_{4}, 5 \mathrm{mmol} / 1 \mathrm{MgCl}_{2}, \mathrm{pH}=7.2$ ) and further split into two aliquots: One for complex I measurement and the other for $\mathrm{CS}$ and protein analysis.

The CS activity was quantified by coupling the release of CoA from acetyl-CoA with the oxidation of DTNB and the following appearance of TNB at $412 \mathrm{~nm}$ in a colorimetric reaction. Briefly, a reaction mix containing $0.15 \mathrm{mM}$ DTNB, $0.5 \mathrm{mM}$ oxaloacetic acid, and $0.1 \%$ Triton X-100 was prepared and incubated for $2 \mathrm{~min}$ at $30^{\circ} \mathrm{C}$. For the measurement, 5,000 cells were used, the reaction was started by the addition of $0.3 \mathrm{mM}$ acetyl-CoA, and the absorbance was recorded kinetically for $2 \mathrm{~min}$.

The activity of CI is directly proportional to ubiquinone reduction, and the co-occurring $\mathrm{NADH}$ consumption was measured at $340 \mathrm{~nm}$. To ensure that complex I activity was not limited by other complexes of the mitochondrial respiratory chain, specific inhibitors of complex III (antimycin A) and IV (KCN) were used as well as $2 \mu \mathrm{g} / \mathrm{ml}$ rotenone and $10 \mu \mathrm{g} / \mathrm{ml}$ antimycin A, respectively, to obtain the specific $\mathrm{NADH}$ oxidation from complex I. For complex I measurement, three additional freeze/thaw cycles of the sample were

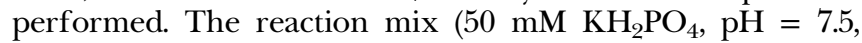
$2.5 \mathrm{mg} / \mathrm{ml} \mathrm{BSA}, 130 \mu \mathrm{M} \mathrm{NADH}$, and $2 \mathrm{mM} \mathrm{KCN)}$ was preincubated for $2 \mathrm{~min}$ at $30^{\circ} \mathrm{C}$, the reaction was started by addition of $65 \mu \mathrm{g} / \mathrm{ml}$ ubiquinone, and the absorbance was measured kinetically for $5 \mathrm{~min}$ at $340 \mathrm{~nm}$.

\section{High-resolution respirometry in permeabilized HeLa cells}

Respiration of fatty acid-supplemented HeLa cells was measured with the Oroboros O2k-FluoRespirometer (Oroboros Instruments, Innsbruck, Austria) and recorded and analyzed using DatLab 7.4 (Oroboros Instruments, Innsbruck, Austria). Two O2ks, with two independent chambers per O2k, were operated in parallel. While one chamber contained cells treated with a fatty acid, the parallel one contained control cells. Instrumental quality control was performed routinely, including daily polarographic oxygen sensor calibrations in mitochondrial respiration medium MiR05 (MiR05-Kit; Oroboros Instruments, Innsbruck, Austria) at $37^{\circ} \mathrm{C}$ and instrumental $\mathrm{O}_{2}$ backgrounds prior to each experimental set. After fatty acid supplementation and cell harvesting (for details, see Materials and methods section), cells were resuspended in MiR05 and added into the O2k chambers via partial volume replacement. During experiments, the oxygen range was kept between air saturation and $70 \mu \mathrm{M}$. Oxygen flux (pmol $\mathrm{O}_{2} /(\mathrm{s}$. $\mathrm{mL})$ ) was automatically corrected for instrumental background in DatLab 7.4, and results were expressed as $\mathrm{O}_{2}$ flow per cell (amol O $2 /(\mathbf{s} \cdot \mathbf{x}))(31-33)$.

The substrate-uncoupler-inhibition-titration (SUIT-008) protocol (34) was used to analyze the oxidative phosphorylation (OXPHOS) capacity of HeLa cells suspended in MiR05. Different pathway control states were induced by the addition of substrates, uncouplers, and inhibitors. ROUTINE respiration was measured in living cells before permeabilization of the plasma membrane with digitonin to enable full access to mitochondria of subsequently titrated substrates and inhibitors. The optimum concentration of digitonin was determined applying the SUIT-010 protocol, yielding an optimum concentration of $0.15 \mu \mathrm{g} / \mathrm{ml}(1.35 \mu \mathrm{M})(35)$. When cells were fully permeabilized, pyruvate $(5 \mathrm{mM})$ and malate $(2 \mathrm{mM})$ were added to support $\mathrm{N}$-linked LEAK respiration followed by the addition of ADP (2.5 mM) to evaluate N-OXPHOS capacity. Cyt $c(10 \mu \mathrm{M})$ was added after the titration of ADP to test the intactness of the mitochondrial outer membrane, followed by addition of glutamate $(10 \mathrm{mM})$. To assess the NS-OXPHOS capacity, succinate $(10 \mathrm{mM})$ was injected to the permeabilized cells. The maximum respiration was experimentally induced by subsequent titrations of the uncoupler CCCP (0.5 $\mu \mathrm{M}$ steps) to reach NS-ET capacity. To inhibit complex I, $0.5 \mu \mathrm{M}$ rotenone was added, reducing respiration to S-ET capacity. Antimycin A $(2.5 \mu \mathrm{M})$ was injected to obtain residual oxygen consumption (Rox).

\section{RESULTS}

For establishing serum-free and lipid-free cell culture, we ordered a customized panserin 401 without lipids (Pan Biotech, Aidenbach, Germany) and used fatty acid supplementation mediated via fatty acid-free BSA (8). To obtain the optimal concentration for lipid supplementation, we determined the cytotoxicity of each supplemented fatty acid using a cell viability assay (CCK-8; Dojindo EU GmbH, Munich, Germany). The $\mathrm{LD}_{50}$ values of the 10 tested fatty acids ranged between $36.6 \mu \mathrm{M}$ for arachidonic acid (AA) and $472.3 \mu \mathrm{M}$ for 
TABLE 1. Viability of HeLa cells in the presence of supplemented fatty acid-BSA conjugates $(n=3)$

\begin{tabular}{llccr}
\hline Fatty Acid & Abbreviation & Name & $\operatorname{Mean}_{(\mathrm{LD} 50)} / \mu \mathrm{M}$ & $\mathrm{SD} / \mu \mathrm{M}$ \\
\hline Oleic acid & OA & $18: 1$ & 472.3 & 3.4 \\
Palmitoleic acid & PoA & $16: 1$ & 285.5 & 5.8 \\
Lauric acid & LaA & $12: 0$ & 225.2 & 3.5 \\
Linoleic acid & LA & $18: 2$ & 182.8 & 0.6 \\
Myristic acid & MA & $14: 0$ & 137.5 & 14.3 \\
Stearic acid & SA & $18: 0$ & 83.6 & 4.4 \\
$\alpha$-Linolenic acid & ALA & $18: 3$ & 66.0 & 2.6 \\
Palmitic acid & PA & $16: 0$ & 61.2 & 6.2 \\
Arachidonic acid & AA & $20: 4$ & 36.6 & 6.8 \\
Docosahexaenoic acid & DHA & $22: 6$ & NA & NA \\
\hline
\end{tabular}

NA, not available.

oleic acid (OA; Table 1). No effect on the viability was observed with DHA in the accessible concentration range of $0-200 \mu \mathrm{M}$, which is in line with other observations $(36,37)$, but contrasts others $(38,39)$, underlining the need to determine viability for each respective model system. Because of the low $\mathrm{LD}_{50}$ for $\mathrm{AA}$, we used $25 \mu \mathrm{M}$ fatty acid as a generally applicable concentration for subsequent supplementation experiments. Interestingly, these relatively low levels of supplemented fatty acids had a positive impact on cell proliferation (supplemental Fig. S1).

To characterize potential lipidomic alterations caused by fatty acid supplementation, HeLa cells were first grown in panserin 401 without lipids for $48 \mathrm{~h}$ followed by $72 \mathrm{~h}$ in the presence of the respective fatty acid-BSA conjugate, and the CL composition was quantified (Fig. 1B) as described (8). Strikingly, each cell culture medium had a unique effect on CL composition (Fig. 1C). This falls in line with our earlier observation that the exact $\mathrm{CL}$ composition is not transcriptionally regulated but rather dependent on the availability of fatty acyls (9). In the same samples, we could observe that the species compositions of phosphatidylethanolamine and phosphatidylcholine were altered in response to the different fatty acid-enriched conditions tested (supplemental Fig. S4). Also in these other phospholipid classes, PUFA supplementation had the strongest influence on the observed lipid profiles.

HeLa cultivated in panserin 401 without lipids can only utilize fatty acids derived from their own de novo biosynthesis, which deprives them of PUFAs, and indeed, the measured CL species were dominated by acyl side chains with a total of only four double bonds (Fig. 1C, left panel). Our fatty acid deconvolution revealed that palmitoleic acid (16:1) and OA (18:1) made up 80\% of the total CL composition, showing an apparent preference of monounsaturated long-chain fatty acids over saturated ones (Fig. 1C, right panel).

A principal component analysis of the CL profiles demonstrates that the effects of supplementation are fatty acid specific with varying intensity (Fig. 1D). The highest degree of incorporation was achieved with linoleic acid (LA; $18: 2)$ to over $70 \%$ of all side chains in favor of 16:1 and 18:1 (Fig. 1C, right panel). Stearic acid (SA;
18:0) supplementation led to an equally strong increase of 18:1 incorporation as treatment with OA suggesting a quick desaturation process of this saturated fatty acid once taken up into the cell. Notably, PA (16:0) was also not incorporated as 16:0 itself but led to a slight increase of 16:1. This likely reflects the fact that PA is the major product of fatty acid biosynthesis and is subsequently elongated and/or desaturated into other nonessential fatty acids such as palmitoleic acid, SA, and OA (40). Myristic acid and AA were both incorporated to a level of $11 \%$ of CL side chains and $\alpha$-linolenic acid (ALA) with 27.5\%. DHA supplementation led to minor changes in CL profile with levels lower than the fatty acyl resolution of the mathematical side-chain modeling approach used (8). In absolute terms, upon LA treatment, a total of $76 \%$ of all CL side chains were altered, followed by ALA (34.2\%), AA (31.4\%), and OA (25.2\%), whereas PA and DHA had the least impact with $8.9 \%$ and $8.3 \%$, respectively (supplemental Fig. S5). Notably, the fatty acyl incorporation was concentration dependent. For example, when PA was supplied in excess at $200 \mu \mathrm{M}-\mathrm{a}$ concentration at which cell viability is already reduced to $10 \%$ within $72 \mathrm{~h}$-the content of PA in CL increases from $4.6 \%$ to $40.4 \%$, when compared with the $25 \mu \mathrm{M}$ PA treatment (supplemental Fig. S6).

LA was the most influential of the 10 supplemented fatty acids, resulting to focus the CL profile around tetralinoleoyl-CL, rather than leading to multiple CL compositions as it was the case for all other supplementations (Fig. 1B). The fraction of LA content in CL side chains however plateaus at $75 \%$ after 4 days of treatment with $25 \mu \mathrm{M}$ (Fig. 1E). This is in good agreement with tetralinoleoyl-CL being the most abundant molecular CL species in most mammalian tissues including heart and muscle (9). In contrast, ALA only incorporated half as frequently when being supplemented in the same concentration, highlighting a clear selectivity for LA. To check how strong the influence of a competing fatty acid was in context of dual mixtures, LA was supplemented with increasing amounts of OA (Fig. 1F). The result was a quenching of LA incorporation, but a significant molar surplus of OA (more than 3 -fold) was needed to achieve a $50 \%$ reduction.

As our cell culture model is a proliferating system, we tested whether LA remains accumulated in CLs after its removal in the medium or if it is readily exchanged with other fatty acids in three different lipid exchange experiments (Fig. 2A). Therefore, cells were preconditioned with lipid-free (without; Fig. 2B, upper panels), OA (Fig. 2B, center panels), and ALA containing medium (Fig. 2B, bottom panels), which was then exchanged for LA containing medium and vice versa. The content of the respective fatty acyls in CL was then tracked over time by means of LC-MS/MS.

We observed a rapid LA incorporation in all three preconditioned scenarios, which was accompanied by a replacement of other fatty acyls including 18:1 and, when present, 18:3 (Fig. 2B). Quantitatively, these effects 


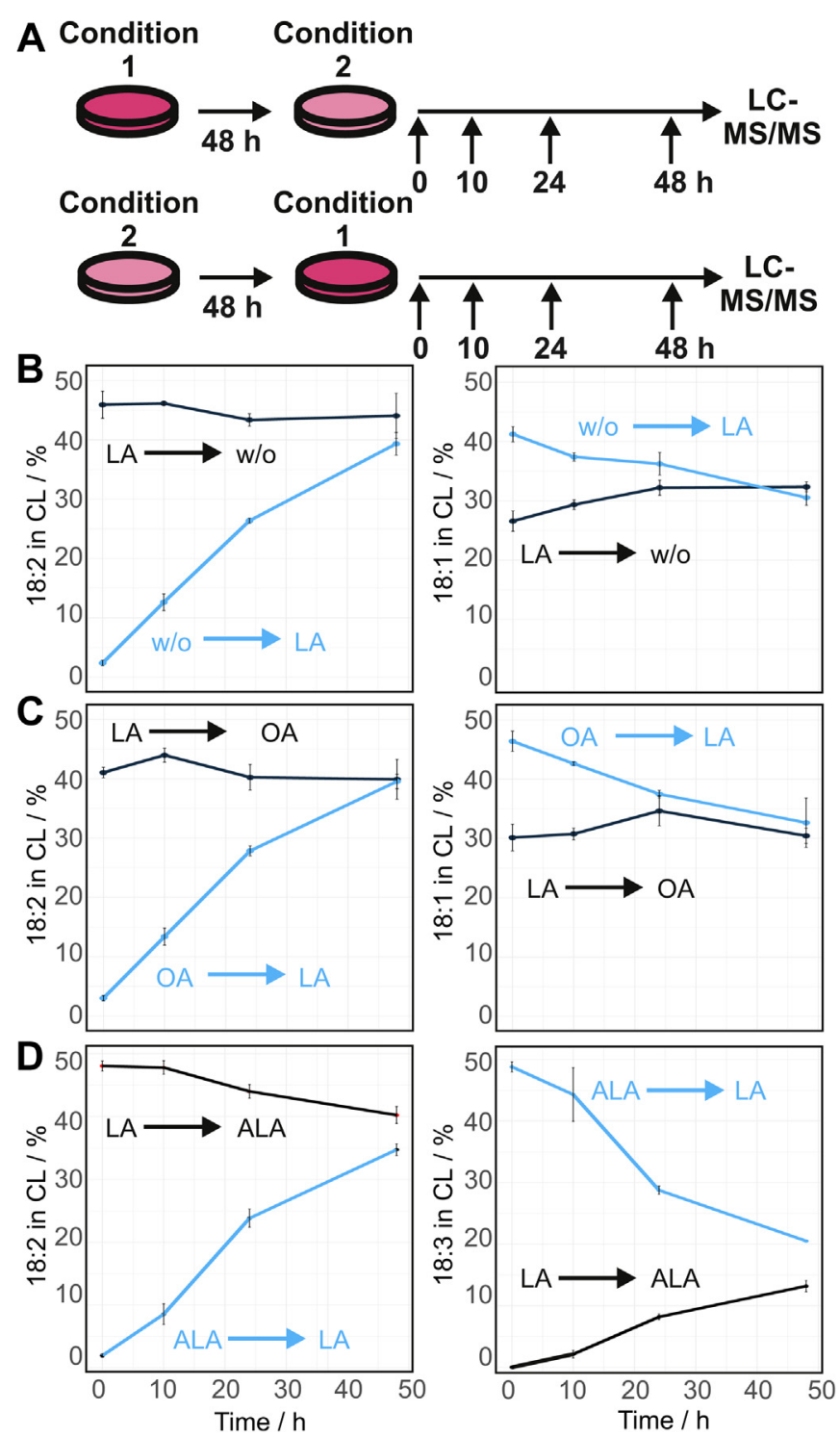

Fig. 2. LA incorporation is preferred even if other FAs are available. A: Schematic depiction of lipid exchange cell culture experiments. B: Panserin 401 without and LA lipid exchange: While the amount of $18: 2$ increased from $2.5 \%$ to $38 \%, 18: 1$ decreased from $42 \%$ to $31 \%$ within $48 \mathrm{~h}$ upon adding $25 \mu \mathrm{MLA}$. In reversed conditions, the amount of 18:2 dropped from $46 \%$ to $44 \%$ and $18: 1$ increased from $27 \%$ to $32 \%$. Thus, $18: 2$ is relatively slowly exchanged in LA-depleted conditions. C: LA and OA exchange: In LA-pretreated conditions, neither 18:2 nor 18:1 content was affected after replacing LA with OA within $48 \mathrm{~h}$. In contrast, 18:1 content decreased from $46 \%$ to $33 \%$ after OA to LA replacement, whereas LA content increased from $3 \%$ to $40 \%$ in the same time. Thus, 18:2 has a lower turnover rate in CL than 18:1. D: LA and ALA exchange: LA content decreased from $47 \%$ to $40 \%$ after ALA replacement, whereas ALA increased from $0 \%$ to $13 \%$ in $48 \mathrm{~h}$. However, in reverse experiment conditions, LA increased from $3 \%$ to $34 \%$, whereas ALA dropped from $48 \%$ to $21 \%$.

were slightly less pronounced when exchanging LA and ALA with each other. Upon LA withdrawal, however, the fraction of linoleoyl side chains in CLs remained largely stable within the period of $48 \mathrm{~h}$. This effect was again least pronounced in the context of ALA. This was surprising for this proliferating system, since at least a dilution factor of two would in theory be expected. This leads to the assumption that other cellular LA reservoirs are first depleted before significant changes occur in the mitochondrial CL composition. Together, this demonstrates that the CL side-chain composition is highly dependent on the availability of fatty acids. Thereby, the CL profile does not directly mirror the available fatty acyls, but the profile is modulated and altered by intrinsic specificities and preferences, especially for LA and to a lesser degree also for ALA.

Next, we used this lipid-free and serum-free model system to address the question how the changes in CL composition affect mitochondrial function. Previously, we demonstrated that supplementation of cultured cells with heart lipid extracts led to a more heart-like CL profile as well as an increase of CI activity (8). Although the heart lipid extract used was composed to a considerable extent of bound and free fatty acids, and an increased proportion of this can be assumed to be LA, it could not be specified whether both effects were caused by exactly the same compound contained in the mixture. Analyzing the impact of single fatty acid-BSA treatments allowed us to determine the individual effect of changing the lipid environment on mitochondria. The enzyme activity of the inner mitochondrial membrane-localized CI was measured after 4 days of fatty acid treatment and reaching a stable CL composition. The enzymatic activity of the matrixlocalized CS served as control.

We observed that the CI activity followed a fatty acid supplement-dependent behavior. Heart lipid supplemented cells were characterized by an increased CI activity compared with controls, whereas CS was unaltered, leading to an increase in CI/CS ratio (Fig. 3A). When supplementing individual fatty acids, the highest specific activity was found in cells supplemented with $25 \mu \mathrm{M}$ LA. Equally high rates were also observed for the combined treatment of $25 \mu \mathrm{M}$ LA with $25 \mu \mathrm{M}$ SA. The latter, when supplemented alone, caused the lowest CI activity, whereas the effect caused by the combinatorial treatment appeared to be driven by LA alone. Both LA containing conditions resulted in specific activities significantly higher compared with treatment with the saturated fatty acids, PA and SA. In contrast, no significant changes between any of the measured conditions were detected regarding CS activity (Fig. 3B), which was determined from the same cell homogenates. Thus, LA stimulated CI activity in permeabilized HeLa cells irrespective of it being supplemented alone or in combination. This effect is in line with earlier observations made for the treatment of HeLa cells with whole heart lipid extracts (8).

This was followed up by testing whether the observed alteration of biochemical properties also affects mitochondrial respiration in HeLa cells. Thus, $\mathrm{O}_{2}$ consumption of differentially supplemented cells 
A Respiratory Complex I

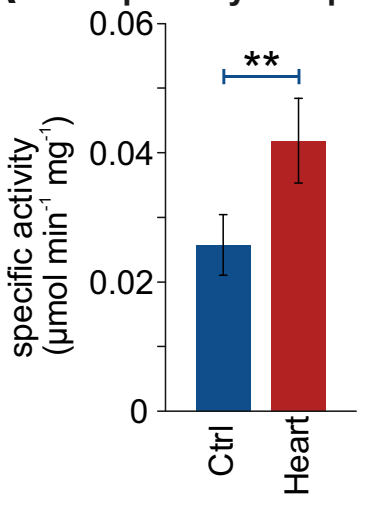

B

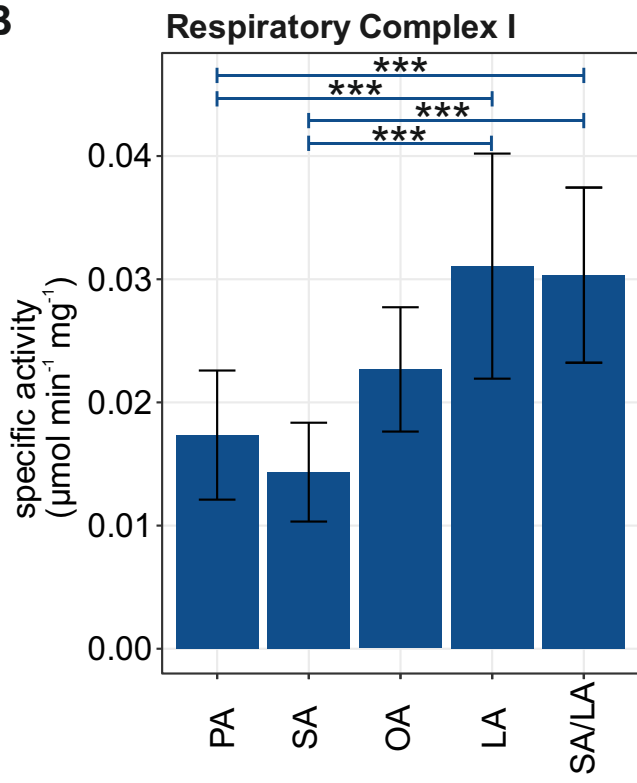

$\mathrm{CI} / \mathrm{CS}$

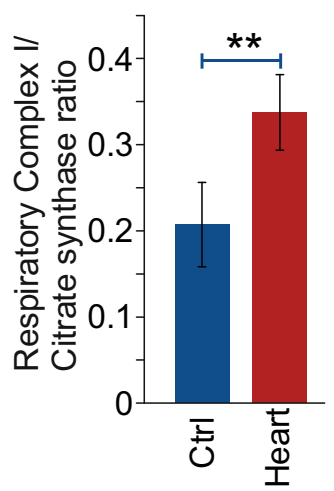

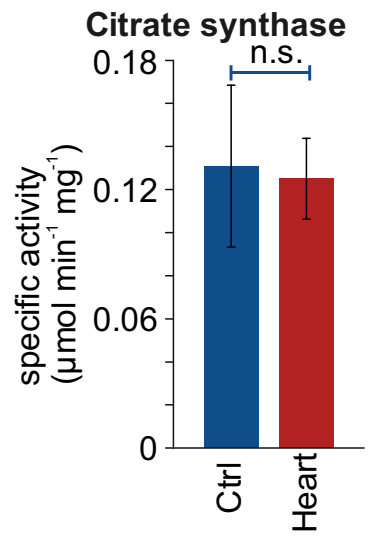

Fig. 3. LA increases the CI activity but not of CS. A: CI and CS activities in heart-lipid supplemented HeLa cells. (3 days, $200 \mu \mathrm{l} / \mathrm{ml}$ heart lipid extract, $\mathrm{n}=6$, mean $\pm \mathrm{SD}$ ). B: CI activity was significantly increased after 4 days in LA supplemented media, including a 1:1 mix of LA and SA. $(\mathrm{n}=10,25 \mu \mathrm{M}$ FA, mean $\pm \mathrm{SD}$, significance was tested by ANOVA and Tukey post hoc correction, $P=7.59 \mathrm{e}-07)$. CS activity was not affected by lipid supplementation in HeLa $(\mathrm{n}=12, P=0.454)$. Significance: $* * * P<0.001$.

was measured in a high-resolution respirometer, following a specific SUIT-008 protocol (34). This allowed us to characterize the behavior of $\mathrm{N}$ - and $\mathrm{S}$ linked pathways (NADH-dependent via CI and succinate-dependent via respiratory complex II) of the mitochondrial electron transfer system in a substrate-dependent manner (Fig. 4). Interestingly, we observed no significant differences of the flux control ratio (normalized for noncoupled electron transfer capacity) in living (ce) and permeabilized cells after digitonin treatment (Dig). The increased biochemical activity of CI upon supplementation of unsaturated fatty acids (Fig. 3) was apparently not rate limiting for fueling OXPHOS in cells when stimulated with NADH-linked substrates and succinate. Of note, we detected a trend toward reduced flux control ratios of N-linked OXPHOS in cells treated with saturated fatty acids (Fig. 3, ADP, c: cyt $c$, G: glutamate). The same behavior was also observed on the level of specific fluxes (supplemental Fig. S7).

\section{DISCUSSION}

The acyl side-chain composition of CL is highly diverse not only in different organisms but also within tissues of the same organism $(8,9,30)$. The postbiosynthetic enzymatic remodeling process of CL plays an important physiological role for mitochondrial membrane constitution, which can cause severe mitochondrial dysfunction, for example, when impaired as in the case of Barth syndrome (41). It is postulated that the diversity of CL is largely driven by two processes, i) the mitochondrial inner membrane's intrinsic strive to minimize free energy, which is strongly shaped by the protein-crowded environment in the membrane (42) and ii) the set of fatty acyls available for CL remodeling that determines the local free energy minimum that can be achieved (9, 43, 44). Taken together, these factors predict a strong dependence of mitochondrial membrane properties on the growth environment, which is with 


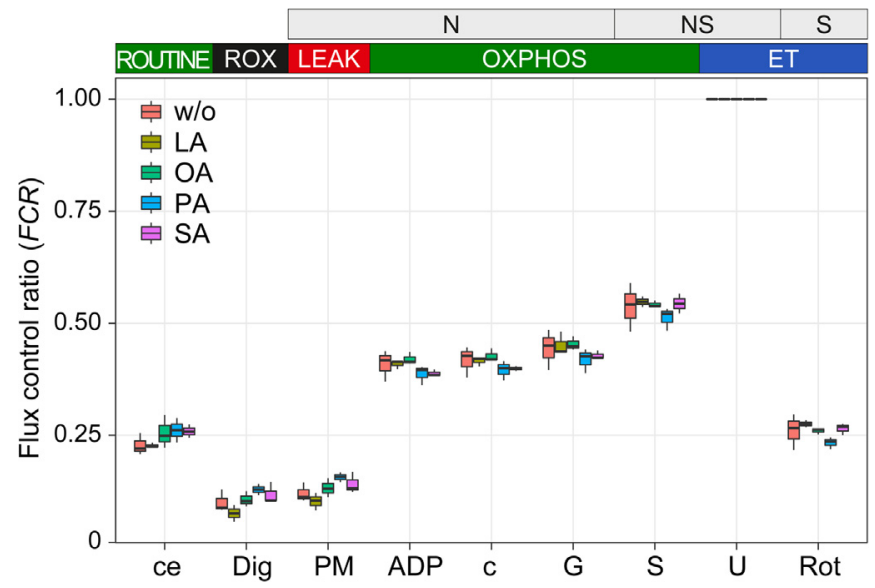

Fig. 4. Fatty acid supplementation does not affect mitochondrial respiration ex vivo. Flux control ratios (FCRs) of living and permeabilized HeLa cultivated in different supplemented media (BSA mock control, LA, OA, PA, and SA) were normally distributed (Shapiro-Wilk's test, $P>0.05$ ) and not significantly altered (ANOVA, $P=0.054, \mathrm{n}=3$ ). FCRs were baseline corrected for residual oxygen consumption $(R o x)$ after inhibiting respiratory complex III with antimycin A. Corresponding $\mathrm{O}_{2}$ flow per cell can be found in supplemental Fig. S7. The header shows the different respiratory states according to the following sequential additions. ADP, ADP to activate OXPHOS; c, cytochrome $c$ for control of intact mitochondrial outer membranes; ce, ROUTINE respiration of living cells; Dig, digitonin for cell permeabilization; $\mathrm{G}$, glutamate as NADH-linked substrate; PM, pyruvate and malate as substrate for NADHdependent OXPHOS; Rot, rotenone to inhibit complex I for determining succinate-dependent respiration; $\mathrm{S}$, succinate to measure NADH and succinate-dependent OXPHOS; U, CCCP to dissipate the membrane potential to reach full electron transfer capacity.

regard to cultured cells defined by the medium and culturing conditions chosen.

As demonstrated in this study, by testing the impact of a range of fatty acid supplements in cell culture, the CL compositions as well as the CI activities respond to changes in the lipid content and composition in the medium. While the content of LA in human plasma is approximately $27 \%$ of free fatty acids, with total PUFA levels of $35-40 \%(45,46)$, the typical lipid content of FBS mainly includes saturated and monounsaturated fatty acids with an LA fraction of as little as 5-8\% $(47,48)$. This results in the general LA-deprived "CL phenotype" found across many different cultured cell lines (8). This does however not reflect the natural diversity of this lipid class. As a result, cells grown under these conditions tend to quickly incorporate large amounts of LA into CLs once this fatty acid is made available (Fig. 1E). Thereby, the degree of change that can be caused by the lipid fraction of the medium depends on the existing composition of the cells (Figs. $1 \mathrm{~F}$ and 2). In this regard, a LA-saturated CL profile is noticeably more robust than other CL states. Not only CL but also other phospholipids respond in a similar way, but following a different specificity pattern (supplemental Fig. S4), which is consistent with findings such as the PUFA-specific response of the phospholipid composition in rat muscles (49).

These observations are in agreement with the finding that the turnover of CL is slower than the turnover of other phospholipids (50-52). We made the observation that the CL state in cells can be highly stable as long as the lipid environment is not altered. However, when switching from LA-depleted to linoleic-rich growth conditions, we find a rapid incorporation of LA into CL (Fig. 2B), reflecting the preference of CL for linoleate incorporation (53). This is reflected in the CL compositions of most tissues, especially heart, where tetralinoleoyl-CL accounts for $\sim 80 \%$ of $\mathrm{CL}$ and is depleted in Barth syndrome $(41,54)$.

The functional importance of a high LA content can be seen in the enzymatic activity assays. While matrix-localized CS was not affected, the membrane bound CI had a significantly higher enzymatic activity (Fig. 3B) highlighting the importance of lipid availability for respiratory complex function. The same result was observed using heart lipid extract for supplementation, which is in line with previous results (8), suggesting that LA could be a major determining factor for this increase. However, when analyzed in the context of living and permeabilized cells, these biochemical differences are compensated for and lead to a balanced respiratory behavior (Fig. 4). If respiratory complex I activity presents a threshold effect with a low flux control coefficient, an increased complex I activity is not expected to be captured by an increased NADH-linked pathway flux (55). Alternatively, this effect could be caused by antagonistic additivities of the fluxes in the convergent pathways of OXPHOS (32). An increased penetrance of the biochemical effects on cellular respiration could be expected under genuine physiological conditions such as in energy demanding tissues, indicating the limitations of the presently used cell culture model. In addition, fatty acids exert a distinct impact on the regulation of gene expression, thus providing another route to modulate the metabolic network and energy metabolism (56).

Our observations open up a multitude of possibilities by which the medium can influence the mitochondrial CLs, which are in line with the recognized unnatural lipid profile in cultured cells (57). These include, for example, batch effects caused by changes in the serum lipid composition. Also differences in the fatty acid depletion rates from the medium could lead to altered CL profiles (58). This would, for example, be the case if cells with differing growth rates are compared and LA in the medium becomes limiting for the faster growing cell line. The same also applies to chemically defined media. Often precise information about their 
composition is not made public, especially with regard to the lipids used $(6,54,59,60)$. Of course, this renders it noticeably more difficult to assess to what extent the medium used is suitable for the desired cellular model system. In an ideal situation, cultivated cells could utilize the same sources and compositions of lipids as in their organism of origin. For CL, one of the main factors is the LA content, whereas other lipid classes might be more responsive to different sets of fatty acids (9).

Another important factor is the concentration with which lipids are supplied to cultured cells. Here, we chose $25 \mu \mathrm{M}$ for all supplementation experiments as this was a concentration that generated completely viable cells, even for AA (Table 1). Already this relatively low concentration strongly affected the side-chain composition of CLs (Fig. 1C). In contrast, other studies use higher concentrations ranging up to $500 \mu \mathrm{M}(61,62)$. Such levels can cause increased apoptosis, cell death, and the induction of endoplasmic reticulum stress (63). Not every cell culture system easily tolerates such high loads of fatty acids. Concentrations of fatty acids that impair cell viability should be avoided unless this is the exact aim of the experiment. In any case, it is advisable to evaluate viability ranges not only for a given cell line in general but also in combination with its respective growth medium. Importantly, it should be noted that the observed behavior could not be transferred directly from one fatty acid to another (compare Table 1).

In a similar manner, also the solubility of fatty acids is variable, which affects the applicability for complex formation with BSA. This manifests itself by the fact that the formation of different fatty acid BSA conjugates (63) correlates with their respective melting points. Unsaturated fatty acids can be solubilized with BSA in higher concentrations than their saturated counterparts (64). Attempting to reach particularly high fatty acid concentrations could lead to increased levels of free fatty acids, which in turn can induce different physiological effects in the cell (65).

Lipids added to the cell culture medium clearly also contribute to cellular energy metabolism and anabolic requirements. We observed slightly increased growth rates of cells in the presence of low concentrations of fatty acid-BSA conjugates, irrespective of the type of fatty acid supplied (supplemental Fig. S1). Such a behavior was absent in fatty acid-free BSA mock controls. It can be assumed that these effects are particularly relevant if the medium used is otherwise lipid free, as is the case in this study. However, the variable impact of different fatty acid supplements on core mitochondrial functions as shown in Fig. 3 is independent of this general behavior with regard to cell proliferation and viability.

In conclusion, the (lipid) composition of cell culture media strongly affects not only mitochondrial phospholipid composition but also mitochondrial functions.
Supplementation, particularly with the essential LA, has a significant effect on mitochondrial activity and could be used to more realistically mimic mammalian tissue in cell culture.

\section{Data availability}

Mass spectrometric raw data and extracted lipid profiles are deposited as Mendeley Dataset (https://doi. org/10.17632/6pdcrm5p6k.1). Scripts for data analysis can be shared upon request (Markus A. Keller, markus. keller@i-med.ac.at).jir

\section{Supplemental data}

This article contains supplemental data.

\section{Author contributions}

J. Z. and M. A. K. conceptualization; J. K. and M. A. K. methodology; J. K. and M. A. K. software; G. O., M.-L. E., Y. W., G. L., and J. K. formal analysis; G. O., M.-L. E., Y. W., K. L., G. L., and L. H. D. C. investigation; K. L., H. H. L., E. G., and S. D. resources; M.-L. E. and J. K. data curation; G. O. and M. A. K. writing-original draft; G. O., M-L. E., Y. W., K. L., G. L., J. K., L. H. D. C., H. H. L., E. G., S. D., J. Z., and M. A. K. writingreview and editing; G. O., M.-L. E., Y. W., and M. A. K. visualization; E. G., S. D., J. Z., and M. A. K. supervision; E. G. and M. A. K. funding acquisition.

Author ORCIDs

Gregor Oemer (10 https://orcid.org/0000-0002-3457-0955

Yvonne Wohlfarter (i) https://orcid.org/0000-0002-09889411

Katharina Lackner (i) https://orcid.org/0000-0003-32609139

Luiza H.D. Cardoso (10 https://orcid.org/0000-0001-63929229

Herbert H. Lindner (D) https://orcid.org/0000-0003-12629976

Sandrine Dubrac (10) https://orcid.org/0000-0002-2936-8488 Johannes Zschocke (D) https://orcid.org/0000-0002-00468274

Markus A. Keller (1) https://orcid.org/0000-0002-8654-9920

Funding and additional information

This work was supported by the Austrian Science Fund (FWF) project P33333 (to M. A. K.) and the Austrian Research Promotion Agency (FFG, \#878654, to M. A. K. and E. G.). G. O. was supported by a "Stipendium der Monatshefte für Chemie" of the Austrian Academy of Sciences.

Conflict of interest

The authors declare that they have no conflicts of interest with the contents of this article.

\section{Abbreviations}

AA, arachidonic acid; ALA, $\alpha$-linolenic acid; CCK-8, Cell Counting Kit-8; CL, cardiolipin; CS, citrate synthase; cyt $c$, cytochrome $c$, FA, fatty acid; LA, linoleic acid; OA, oleic acid; OXPHOS, oxidative phosphorylation; PA, palmitic acid; CI, 
respiratory complex I; SA, stearic acid; SUIT, substrate-uncoupler-inhibition-titration.

Manuscript received January 12, 2021, and in revised from August 18, 2021. Published, JLR Papers in Press, August 24, 2021, https://doi.org/10.1016/j.j1r.2021.100111

\section{REFERENCES}

1. Maqsood, M. I., Matin, M. M., Bahrami, A. R., and Ghasroldasht, M. M. (2013) Immortality of cell lines: challenges and advantages of establishment. Cell Biol. Int. 37, 1038-1045

2. Lucey, B. P., Nelson-Rees, W. A., and Hutchins, G. M. (2009) Henrietta lacks, HeLa cells, and cell culture contamination. Arch. Pathol. Lab. Med. 133, 1463-1467

3. Eagle, H. (1955) Nutrition needs of mammalian cells in tissue culture. Science. 122, 501-504

4. Dulbecco, R., and Freeman, G. (1959) Plaque production by the polyoma virus. Virology. 8, 396-397

5. McKee, T. J., and Komarova, S. V. (2017) Is it time to reinvent basic cell culture medium? Am. J. Physiol. Cell Physiol. 312, C624-C626

6. van der Valk, J., Brunner, D., De Smet, K., Fex Svenningsen, A., Honegger, P., Knudsen, L. E., Lindl, T., Noraberg, J., Price, A., Scarino, M. L., and Gstraunthaler, G. (2010) Optimization of chemically defined cell culture media-replacing fetal bovine serum in mammalian in vitro methods. Toxicol. In Vitro. 24, 1053-1063

7. Hulbert, A. J., Turner, N., Storlien, L. H., and Else, P. L. (2005) Dietary fats and membrane function: implications for metabolism and disease. Biol. Rev. Camb. Philos. Soc. 80, 155-169

8. Oemer, G., Lackner, K., Muigg, K., Krumschnabel, G., Watschinger, K., Sailer, S., Lindner, H., Gnaiger, E., Wortmann, S. B., Werner, E. R., Zschocke, J., and Keller, M. A. (2018) Molecular structural diversity of mitochondrial cardiolipins. Proc. Natl. Acad. Sci. U. S. A. 115, 4158-4163

9. Oemer, G., Koch, J., Wohlfarter, Y., Alam, M. T., Lackner, K., Sailer, S., Neumann, L., Lindner, H. H., Watschinger, K., Haltmeier, M., Werner, E. R., Zschocke, J., and Keller, M. A. (2020) Phospholipid acyl chain diversity controls the tissue-specific assembly of mitochondrial cardiolipins. Cell Rep. 30, 4281-4291.e4

10. Sniezek, O. L., Anzmann, A. F., Claypool, S. M., and Vernon, H. J. (2020) Cardiolipin's remodeling rules revealed: the role of the cellular lipidome. Cell Rep. 30, 3949-3950

11. Daum, G. (1985) Lipids of mitochondria. Biochim. Biophys. Acta. 822, $1-42$

12. De Kroon, A. I. P. M., Dolis, D., Mayer, A., Lill, R., and De Kruijff, B. (1997) Phospholipid composition of highly purified mitochondrial outer membranes of rat liver and Neurospora crassa. Is cardiolipin present in the mitochondrial outer membrane? Biochim. Biophys. Acta. 1325, 108-116

13. Ikon, N., and Ryan, R. O. (2017) Cardiolipin and mitochondrial cristae organization. Biochim. Biophys. Acta Biomembr. 1859, 1156-1163

14. Kameoka, S., Adachi, Y., Okamoto, K., Iijima, M., and Sesaki, H. (2018) Phosphatidic acid and cardiolipin coordinate mitochondrial dynamics. Trends Cell Biol. 28, 67-76

15. Ban, T., Ishihara, T., Kohno, H., Saita, S., Ichimura, A., Maenaka, K., Oka, T., Mihara, K., and Ishihara, N. (2017) Molecular basis of selective mitochondrial fusion by heterotypic action between OPA1 and cardiolipin. Nat. Cell Biol. 19, 856-863

16. Bazán, S., Mileykovskaya, E., Mallampalli, V. K. P. S., Heacock, P., Sparagna, G. C., and Dowhan, W. (2013) Cardiolipin-dependent reconstitution of respiratory supercomplexes from purified Saccharomyces cerevisiae complexes III and IV.J. Biol. Chem. 288, 401-411

17. Pfeiffer, K., Gohil, V., Stuart, R. A., Hunte, C., Brandt, U., Greenberg, M. L., and Schägger, H. (2003) Cardiolipin stabilizes respiratory chain supercomplexes. J. Biol. Chem. 278, 52873-52880

18. Chatzispyrou, I. A., Guerrero-Castillo, S., Held, N. M., Ruiter, J. P. N., Denis, S. W., IJlst, L., Wanders, R. J., van Weeghel, M., Ferdinandusse, S., Vaz, F. M., Brandt, U., and Houtkooper, R. H. (2018) Barth syndrome cells display widespread remodeling of mitochondrial complexes without affecting metabolic flux distribution. Biochim. Biophys. Acta Mol. Basis Dis. 1864, 3650-3658
19. Schug, Z. T., and Gottlieb, E. (2009) Cardiolipin acts as a mitochondrial signalling platform to launch apoptosis. Biochim. Biophys. Acta. 1788, 2022-2031

20. Petrosillo, G., Casanova, G., Matera, M., Ruggiero, F. M., and Paradies, G. (2006) Interaction of peroxidized cardiolipin with rat-heart mitochondrial membranes: induction of permeability transition and cytochrome c release. FEBS Lett. 580, 6311-6316

21. Chu, C. T., Ji, J., Dagda, R. K., Jiang, J. F., Tyurina, Y. Y., Kapralov, A. A., Tyurin, V. A., Yanamala, N., Shrivastava, I. H., Mohammadyani, D., Qiang Wang, K. Z., Zhu, J., Klein-Seetharaman, J., Balasubramanian, K., Amoscato, A. A., et al. (2013) Cardiolipin externalization to the outer mitochondrial membrane acts as an elimination signal for mitophagy in neuronal cells. Nat. Cell Biol. 15, $1197-1205$

22. Li, X. X., Tsoi, B., Li, Y. F., Kurihara, H., and He, R. R. (2015) Cardiolipin and its different properties in mitophagy and apoptosis. J. Histochem. Cytochem. 63, 301-311

23. Paradies, G., Paradies, V., De Benedictis, V., Ruggiero, F. M., and Petrosillo, G. (2014) Functional role of cardiolipin in mitochondrial bioenergetics. Biochim. Biophys. Acta. 1837, 408-417

24. Claypool, S. M., and Koehler, C. M. (2012) The complexity of cardiolipin in health and disease. Trends Biochem. Sci. 37, 32-41

25. McGree, C. D., Lieberman, P., and Greenwood, C. E. (1996) Dietary fatty acid composition induces comparable changes in cardiolipin fatty acid profile of heart and brain mitochondria. Lipids. 31, 611-616

26. Belikova, N. A., Vladimirov, Y. A., Osipov, A. N., Kapralov, A. A., Tyurin, V. A., V Potapovich, M., V Basova, L., Peterson, J., V Kurnikov, I., and Kagan, V. E. (2006) Peroxidase activity and structural transitions of cytochrome $\mathrm{c}$ bound to cardiolipincontaining membranes. Biochemistry. 45, 4998-5009

27. Folch, J., Lees, M., and Sloane Stanley, G. H. (1957) A simple method for the isolation and purification of total lipides from animal tissues. J. Biol. Chem. 226, 497-509

28. Pluskal, T., Castillo, S., Villar-Briones, A., and Orešič, M. (2010) MZmine 2: modular framework for processing, visualizing, and analyzing mass spectrometry-based molecular profile data. $B M C$ Bioinformatics. 11, 395

29. Team, R. (2015) R development core team. RA Lang. Environ. Stat. Comput. 55, 275-286

30. Koch, J., Lackner, K., Wohlfarter, Y., Sailer, S., Zschocke, J., Werner, E. R., Watschinger, K., and Keller, M. A. (2020) Unequivocal mapping of molecular ether lipid species by LC-MS/ MS in plasmalogen-deficient mice. Anal. Chem. 92, 11268-11276

31. Gnaiger, E. (2020) Mitochondrial pathways and respiratory control an introduction to OXPHOS analysis. Bioenerg. Commun. 2, $1-112$

32. Gnaiger, E., and MitoEAGLE Task Group (2020) Mitochondrial physiology. Bioenerg. Commun. 1, 1-44

33. Gnaiger, E. (2008) Polarographic oxygen sensors, the oxygraph, and high-resolution respirometry to assess mitochondrial function. In Drug-Induced Mitochondrial Dysfunction. J. Dykens and Y. Will, editors. John Wiley \& Sons, Inc, Hoboken, NJ, 325-352

34. Lemieux, H., Blier, P. U., and Gnaiger, E. (2017) Remodeling pathway control of mitochondrial respiratory capacity by temperature in mouse heart: electron flow through the Q-junction in permeabilized fibers. Sci. Rep. 7, 2840

35. Doerrier, C., Garcia-Souza, L. F., Krumschnabel, G., Wohlfarter, Y., Mészáros, A. T., and Gnaiger, E. (2018). In Methods in Molecular Biology. Humana Press Inc, Totowa, NJ, 31-70

36. Mansara, P. P., Deshpande, R. A., Vaidya, M. M., and Kaul-Ghanekar, R. (2015) Differential ratios of omega fatty acids (AA/ EPA+DHA) modulate growth, lipid peroxidation and expression of tumor regulatory MARBPs in breast cancer cell lines MCF7 and MDA-MB-231. PLoS One. 10, e0136542

37. Song, J., Li, C., Lv, Y., Zhang, Y., Amakye, W. K., and Mao, L. (2017) DHA increases adiponectin expression more effectively than EPA at relative low concentrations by regulating PPAR $\gamma$ and its phosphorylation at Ser273 in 3T3-L1 adipocytes. Nutr. Metab. 14, 52

38. Sangeetha Sagar, P., Das, U. N., Koratkar, R., Ramesh, G., Padma, M., and Sravan Kumar, G. (1992) Cytotoxic action of cisunsaturated fatty acids on human cervical carcinoma (HeLa) cells: relationship to free radicals and lipid peroxidation and its modulation by calmodulin antagonists. Cancer Lett. 63, 189-198

39. Wang, X., Zhao, X., Mao, Z. Y., Wang, X. M., and Liu, Z. L. (2003) Neuroprotective effect of docosahexaenoic acid on glutamate- 
induced cytotoxicity in rat hippocampal cultures. Neuroreport. 14, 395-401

40. Ntambi, J. M., and Miyazaki, M. (2004) Regulation of stearoyl-CoA desaturases and role in metabolism. Prog. Lipid Res. 43, 91-104

41. Schlame, M., and Ren, M. (2006) Barth syndrome, a human disorder of cardiolipin metabolism. FEBS Lett. 580, 5450-5455

42. Xu, Y., Anjaneyulu, M., Donelian, A., Yu, W., Greenberg, M. L. Ren, M., Owusu-Ansah, E., and Schlame, M. (2019) Assembly of the complexes of oxidative phosphorylation triggers the remodeling of cardiolipin. Proc. Natl. Acad. Sci. U. S. A. 166, 11235-11240

43. Schlame, M., Ren, M., Xu, Y., Greenberg, M. L., and Haller, I. (2005) Molecular symmetry in mitochondrial cardiolipins. Chem. Phys. Lipids. 138, 38-49

44. Abe, M., Sawada, Y., Uno, S., Chigasaki, S., Oku, M., Sakai, Y., and Miyoshi, H. (2017) Role of acyl chain composition of phosphatidylcholine in tafazzin-mediated remodeling of cardiolipin in liposomes. Biochemistry. 56, 6268-6280

45. Iuliano, L., Monticolo, R., Straface, G., Zullo, S., Galli, F., Boaz, M. and Quattrucci, S. (2009) Association of cholesterol oxidation and abnormalities in fatty acid metabolism in cystic fibrosis. Am. J. Clin. Nutr. 90, 477-484

46. Coste, T. C., Deumer, G., Reychler, G., Lebecque, P., Wallemacq, P., and Leal, T. (2008) Influence of pancreatic status and sex on polyunsaturated fatty acid profiles in cystic fibrosis. Clin. Chem. 54, 388-395

47. Villard, P. H., Barlesif, F., Armand, M., Dao, T. M. A., Pascussi, J M., Fouchier, F., Champion, S., Dufour, C., Giniès, C., Khalil, A., Amiot, M. J., Barra, Y., and Seree, E. (2011) CYP1A1 induction in the colon by serum: involvement of the PPAR $\alpha$ pathway and evidence for a new specific human PPRE $\alpha$ site. PLoS One. 6 , e14629

48. Gregory, M. K., King, H. W., Bain, P. A., Gibson, R. A., Tocher, D. R., and Schuller, K. A. (2011) Development of a fish cell culture model to investigate the impact of fish oil replacement on lipid peroxidation. Lipids. 46, 753-764

49. Abbott, S. K., Else, P. L., and Hulbert, A. J. (2010) Membrane fatty acid composition of rat skeletal muscle is most responsive to the balance of dietary n-3 and n-6 PUFA. Br. J. Nutr. 103, 522-529

50. Xu, Y., Phoon, C. K. L., Berno, B., D'Souza, K., Hoedt, E., Zhang, G. Neubert, T. A., Epand, R. M., Ren, M., and Schlame, M. (2016) Loss of protein association causes cardiolipin degradation in Barth syndrome. Nat. Chem. Biol. 12, 641-647

51. Wahjudi, P. N., Yee, J. K., Martinez, S. R., Zhang, J., Teitell, M. Nikolaenko, L., Swerdloff, R., Wang, C., and Lee, W. N. P. (2011)
Turnover of nonessential fatty acids in cardiolipin from the rat heart. J. Lipid Res. 52, 2226-2233

52. Schlame, M., and Greenberg, M. L. (2017) Biosynthesis, remodeling and turnover of mitochondrial cardiolipin. Biochim. Biophys. Acta. 1862, 3-7

53. Bradley, R. M., Stark, K. D., and Duncan, R. E. (2016) Influence of tissue, diet, and enzymatic remodeling on cardiolipin fatty acyl profile. Mol. Nutr. Food Res. 60, 1804-1818

54. Sparagna, G. C., Chicco, A. J., Murphy, R. C., Bristow, M. R. Johnson, C. A., Rees, M. L., Maxey, M. L., McCune, S. A., and Moore, R. L. (2007) Loss of cardiac tetralinoleoyl cardiolipin in human and experimental heart failure. J. Lipid Res. 48, 1559-1570

55. Rossignol, R., Faustin, B., Rocher, C., Malgat, M., Mazat, J. P., and Letellier, T. (2003) Mitochondrial threshold effects. Biochem. J. 370, 751-762

56. Georgiadi, A., and Kersten, S. (2012) Mechanisms of gene regulation by fatty acids. Adv. Nutr. 3, 127-134

57. Else, P. L. (2020) The highly unnatural fatty acid profile of cells in culture. Prog. Lipid Res. 77, 101017

58. Keller Markus, A. (2021) Interpreting phospholipid and cardiolipin profiles in rare mitochondrial diseases. Curr. Opin. Syst. In press

59. Bhatia, S. (2019). In Introduction to Pharmaceutical Biotechnology (Vol. 3,. MJP Publisher, Tamil Nadu, India, 218

60. Pan, X., Streefland, M., Dalm, C., Wijffels, R. H., and Martens, D. E. (2017) Selection of chemically defined media for CHO cell fed-batch culture processes. Cytotechnology. 69, 39-56

61. Schilling, J. D., Machkovech, H. M., He, L., Sidhu, R., Fujiwara, H. Weber, K., Ory, D. S., and Schaffer, J. E. (2013) Palmitate and lipopolysaccharide trigger synergistic ceramide production in primary macrophages. J. Biol. Chem. 288, 2923-2932

62. Singh, R., Kaushik, S., Wang, Y., Xiang, Y., Novak, I., Komatsu, M., Tanaka, K., Cuervo, A. M., and Czaja, M. J. (2009) Autophagy regulates lipid metabolism. Nature. 458, 1131-1135

63. Oliveira, A. F., Cunha, D. A., Ladriere, L., Igoillo-Esteve, M. Bugliani, M., Marchetti, P., and Cnop, M. (2015) In vitro use of free fatty acids bound to albumin: a comparison of protocols. Biotechniques. 58, 228-233

64. Alsabeeh, N., Chausse, B., Kakimoto, P. A., Kowaltowski, A. J., and Shirihai, O. (2018) Cell culture models of fatty acid overload: problems and solutions. Biochim. Biophys. Acta Mol. Cell Biol. Lipids. 1863, 143-151

65. Kimura, I., Ichimura, A., Ohue-Kitano, R., and Igarashi, M. (2020) Free fatty acid receptors in health and disease. Physiol. Rev. 100, $171-210$ 